PAPER

Magnetization and ferromagnetic resonance in a Fe/Gd multilayer: experiment and modelling

To cite this article: A B Drovosekov et al 2017 J. Phys.: Condens. Matter 29115802

View the article online for updates and enhancements.
Related content

$$
\begin{aligned}
& \text { - Magnetization curves of an Fe/Gd } \\
& \frac{\text { multilayer film calculatedfrom Gd }}{\text { magnetization depth profiles determined }} \\
& \frac{\text { by resonant x-raymagnetic scattering }}{\text { N Hosoito, H Hashizume and N Ishimatsu }} \\
& \text { - Magnetic multilavers: spin configurations. } \\
& \text { excitations and giant magnetoresistance } \\
& \text { R E Camley and R L Stamps } \\
& \text { - In situ ferromagnetic resonance: an } \\
& \text { ultimate tool to investigate the coupling } \\
& \text { inultrathin magnetic films } \\
& \text { J Lindner and K Baberschke }
\end{aligned}
$$

\section{Recent citations}

- The influence of chemical impurities on the
properties of heavy rare-earth metals (Tb.
Dy. Ho): Experimental and theoretical
approaches
Aleksei S. Komlev et al
- Twisted magnetization states and
inhomogeneous resonance modes in a
Fe/Gd ferrimagnetic multilayer
A.B. Drovosekov et al




\title{
Magnetization and ferromagnetic resonance in a Fe/Gd multilayer: experiment and modelling
}

\author{
A B Drovosekov ${ }^{1}$, N M Kreines ${ }^{1}$, A O Savitsky ${ }^{1}$, E A Kravtsov ${ }^{2,3}$, \\ M V Ryabukhina ${ }^{2}$, V V Proglyado ${ }^{2}$ and V V Ustinov ${ }^{2}$ \\ ${ }^{1}$ P. L. Kapitza Institute for Physical Problems RAS, Kosygina St. 2, 119334 Moscow, Russia \\ 2 M. N. Mikheev Institute of Metal Physics UB RAS, S. Kovalevskaya St. 18, 620990 Ekaterinburg, \\ Russia \\ ${ }^{3}$ Ural Federal University, Mira St., 2, 620002 Ekaterinburg, Russia \\ E-mail: drovosekov@kapitza.ras.ru
}

Received 29 September 2016, revised 8 December 2016

Accepted for publication 20 December 2016

Published 3 February 2017

\begin{abstract}
Static and dynamic magnetic properties of a $[\mathrm{Fe}(35 \AA) / \mathrm{Gd}(50 \AA)]_{12}$ superlattice are investigated experimentally in the temperature range 5-295 K using SQUID magnetometery and the ferromagnetic resonance (FMR) technique at frequencies 7-38 GHz. The obtained magnetization curves and FMR spectra are analysed theoretically using numerical simulation on the basis of the effective field model. At every given temperature, both static and resonance experimental data can be approximated well within the proposed model. However, a considerable temperature dependence of the effective field parameter in gadolinium layers has to be taken into account to achieve reasonable agreement with the experimental data in the entire temperature range studied. To describe the peculiarities of experimental FMR spectra, a non-local diffusion-type absorption term in Landau-Lifshitz equations is considered in addition to the Gilbert damping term. Possible reasons for the observed effects are discussed.
\end{abstract}

Keywords: Fe/Gd multilayer, mean-field model, ferromagnetic resonance, spin diffusion

(Some figures may appear in colour only in the online journal)

\section{Introduction}

Magnetic layered structures have been an object of extensive investigations in recent decades. The $\mathrm{Fe} / \mathrm{Gd}$ multilayer is one of the most interesting systems of this sort, demonstrating a rich magnetic phase diagram. Depending on the applied magnetic field $H$ and temperature $T$, different magnetic order types can be realized, including collinear and non-collinear alignment of the ferromagnetic (FM) layers with respect to the external field. In weak magnetic fields, the antiparallel ordering of FM layers prevails, with Fe magnetization oriented either in the direction of the field ('Fe-aligned state') or opposite to the field ('Gd-aligned state'). Due to a large antiferromagnetic (AFM) coupling at the $\mathrm{Fe}-\mathrm{Gd}$ interface and relatively weak FM exchange in gadolinium, a sufficiently strong external magnetic field can initiate essentially non-uniform distribution of magnetization inside Gd layers ('twisted state').

Magnetic properties of Fe/Gd structures have been investigated by a variety of experimental techniques (see reviews $[1,2])$. In particular, the existence of different magnetic states and the possibility of strongly non-uniform distribution of the magnetic moment in Gd layers was clearly demonstrated by the resonant $\mathrm{x}$-ray magnetic reflectometry technique in a set of works [3-7].

To describe magnetic properties of such a complex system theoretically, the mean-field approach is used [8]. In spite of its simplicity, this model predicts all the main features of the system. However, quantitative agreement with experiment is under question. Detailed magnetization data obtained in a wide range of temperatures and magnetic fields are described only qualitatively in the frame of the effective field model [9]. 
The temperature dependence of magnetization in Gd layers was reported to be close to linear [10] which contradicts standard mean-field theory.

The reasons for the discrepancy between the experiment and the mean-field approach could be due to both the structure imperfections of the real Fe/Gd multilayer and insufficient accuracy of the theory. A weak point of the mean-field approximation is the simplified model assumption that the effective exchange field $H_{\mathrm{ex}}$ is connected with the average magnetization $M$ by simple relation $H_{\mathrm{ex}}=\lambda M$, where $\lambda$ is a constant. To improve the effective field model, different approaches were proposed [11]. In particular, the constant coupling approximation [12] leads to a more complex expression for the effective field $H_{\mathrm{ex}}=\Phi(T, M)$ which can be roughly written as $H_{\mathrm{ex}} \approx \lambda(T) M$ with temperature dependent effective field parameter $\lambda(T)$.

In the present work we analyse magnetic properties of a Fe/ Gd superlattice in the frame of the mean field model taking into account a possible dependence of the effective field constant $\lambda$ on temperature. The applicability of the proposed approach is tested via comparison of theoretical modelling results with static magnetization and FMR data obtained experimentally in a wide 5-295 $\mathrm{K}$ temperature range.

Recently, studies of magnetization dynamics in rare-earth/ transition-metal multilayers attracted attention as these materials are considered as candidates for the realization of ultrafast magnetic switching [13]. In particular, the Fe/Gd structure is a possible system of this sort [14]. Nevertheless, to the best of our knowledge, to date there have been no investigations of FMR in Fe/Gd multilayers below room temperature. In the work [15], we studied FMR in $\mathrm{Fe} / \mathrm{Cr} / \mathrm{Gd}$ systems which are essentially different from $\mathrm{Fe} / \mathrm{Gd}$. Indeed, introduction of the $\mathrm{Cr}$ spacer between $\mathrm{Fe}$ and $\mathrm{Gd}$ layers reduces the interlayer coupling significantly. As a result, in contrast to the Fe/Gd structure, the effect of magnetization twist in Gd layers for this system is negligible.

FMR in $\mathrm{Co} / \mathrm{Gd}$ layered structures (similar to $\mathrm{Fe} / \mathrm{Gd}$ multilayers) was studied by several groups. In the work [16], FMR was investigated for trilayer $\mathrm{Gd} / \mathrm{Co}(40 \AA) / \mathrm{Gd}$ structures with very thin (5-7 $\AA$ ) Gd layers. In this situation, only a weak temperature dependent shift of the absorption line was observed which was described qualitatively on the basis of the mean-field calculations. In the works [17, 18], $\mathrm{Co}(30 \AA) / \operatorname{Gd}(75 \AA)$ superlattices with much thicker Gd layers demonstrated much stronger temperature dependence of FMR spectra. Two absorption lines were observed [17]. However, there were no attempts to analyse the data within the framework of the mean-field model. Meanwhile, resonance frequencies in magnetic multilayers are very sensitive to exchange coupling parameters of the system. Thus, a comparison of the experimental FMR data with the results of the mean-field modelling is of interest.

In the present work, we analyse the experimental FMR spectra in the Fe/Gd superlattice on the basis of the effective field approach, paying special attention to the role of magnetic relaxation in FM layers for FMR frequencies and resonance line widths. In addition to the more commonly used local Gilbert damping [16, 19], we analyse a non-local

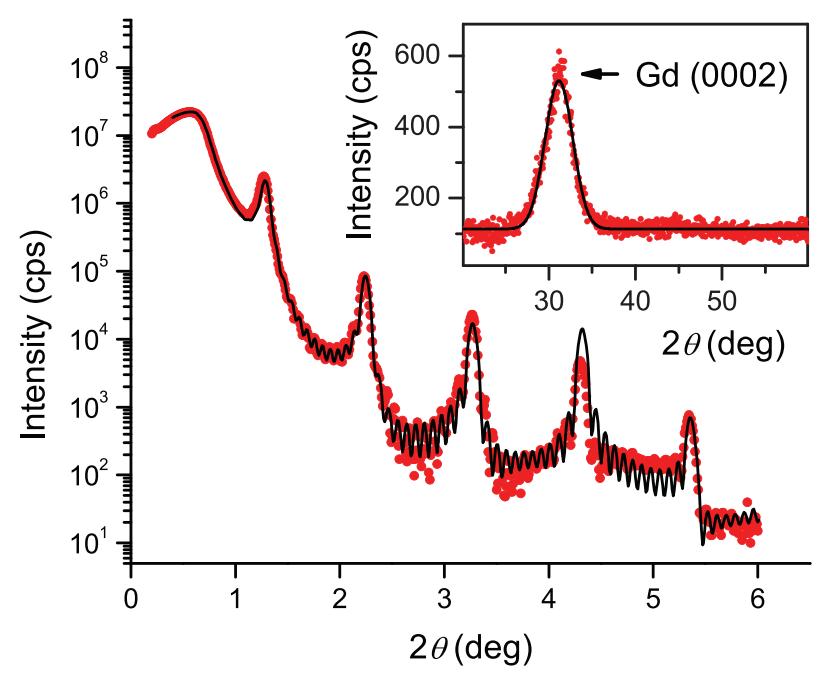

Figure 1. Experimental XRR spectrum of the studied glass $/ \mathrm{Cr}(50 \AA) /[\mathrm{Fe}(35 \AA) / \operatorname{Gd}(50 \AA)]_{12} / \operatorname{Cr}(30 \AA)$ multilayer structure (points) and its approximation (line) with parameters shown in table 1 . The inset demonstrates the experimental XRD spectrum.

Table 1. Parameters of the superlattice extracted from XRR.

\begin{tabular}{lllll}
\hline Layer & & $\begin{array}{l}\text { Density } \\
\left(\mathrm{g} \mathrm{cm}^{-3}\right)\end{array}$ & $\begin{array}{l}\text { Thickness } \\
(\AA)\end{array}$ & $\begin{array}{l}\text { rms roughness } \\
(\AA)\end{array}$ \\
\hline Glass & Substrate & 4.0 & - & 8.8 \\
$\mathrm{Cr}$ & Buffer & 7.1 & 47.0 & 1.0 \\
\hline $\mathrm{Fe}$ & \rceil$_{\times 12}$ & 7.9 & 33.6 & 1.4 \\
$\mathrm{Gd}$ & $」$ & 7.8 & 49.8 & 3.5 \\
\hline $\mathrm{Cr}$ & Cap layer & 7.0 & 20.1 & 4.0 \\
$\mathrm{Cr}_{2} \mathrm{O}_{3}$ & & 5.6 & 18.6 & 9.0 \\
\hline
\end{tabular}

diffusion-type mechanism of magnetic relaxation which is recently discussed in view of its importance for potential development of spintronic devices [20,21].

\section{Sample and experimental techniques}

A multilayer $\left[\mathrm{Fe}\left(t_{\mathrm{Fe}}\right) / \mathrm{Gd}\left(t_{\mathrm{Gd}}\right)\right]_{12}$ structure with nominal FM layer thicknesses $t_{\mathrm{Fe}} \approx 35 \AA$ and $t_{\mathrm{Gd}} \approx 50 \AA$ was deposited using high vacuum magnetron sputtering on a glass substrate with $50 \AA$ thick chromium buffer layer. To prevent oxidation, a $30 \AA$ chromium cap layer was deposited on the top of the structure.

Structural characterization of the sample was performed by $\mathrm{x}$-ray diffraction (XRD) and reflectometry (XRR). The measurements were carried out on a laboratory Empyrean PANalytical diffractometer using $\mathrm{Cu} K_{\alpha}$ radiation. Figure 1 shows the experimental XRR spectrum together with a fitting curve. The structural refinement results are presented in table 1 . We note that the sample has a well-defined layered structure with interfacial root mean square roughness of about 1-2 atomic monolayers. This result is in agreement with [22] where the very sharp interfaces were found in Fe/Gd layered structures with negligible Fe-Gd interdiffusion.

The inset in figure 1 shows the experimental XRD pattern measured in $\theta-2 \theta$ geometry. The XRD spectrum demonstrates 
only one very broad peak corresponding to (0002) hcp Gd reflection. Such a broad peak indicates polycrystalline structure of the grown Gd layers. The corresponding correlation length estimated via the Scherrer equation is about $20 \AA$. We detected no signal from Fe which means that Fe layers are likely to be amorphous when grown in a Fe/Gd multilayer on a glass substrate.

Static magnetization of the sample was investigated in the temperature range $5-295 \mathrm{~K}$ in magnetic fields up to $50 \mathrm{kOe}$, using a conventional SQUID magnetometer Quantum Design MPMS. Magnetic properties of the substrate were measured separately and its contribution was subtracted from the total magnetic moment of the sample.

FMR was studied using a laboratory developed transmission type spectrometer in the range of frequencies $7-37 \mathrm{GHz}$ at temperatures $5-295 \mathrm{~K}$ in magnetic fields up to $10 \mathrm{kOe}$.

In all the experiments, the external magnetic field was applied in the film plane.

\section{Calculation procedure}

\subsection{Static magnetization modelling}

Magnetic characteristics of the superlattice were calculated using the mean field approach. The general idea of the calculation procedure is similar to that described in [2]. Due to a high $T_{\mathrm{C}}$ and a large exchange stiffness of Fe layers, they are considered as homogeneously magnetized up to saturation value $M_{\mathrm{Fe}}^{\mathrm{S}}$ at temperatures under study. To model the magnetization distribution in Gd layers, they are divided into 16 sublayers with atomic scale thickness $t=t_{\mathrm{Gd}} / 16 \approx 3.1 \AA$. The total energy per unit area of the superlattice is given by the sum over all sublayers:

$$
E=\sum_{p=1}^{12} \sum_{l=0}^{16}\left(-t_{p}^{l} \boldsymbol{H} \boldsymbol{M}_{p}^{l}+t_{p}^{l} \frac{K_{p}^{l}}{2}\left(\boldsymbol{M}_{p}^{l}, \boldsymbol{n}\right)^{2}+E_{p}^{l}\right) .
$$

Here $p$ is the index of superlattice period. For every $p$, the index $l=0$ corresponds to the Fe layer with thickness $t_{p}^{0}=t_{\mathrm{Fe}}$ and magnetization vector $\boldsymbol{M}_{p}^{0}\left(M_{p}^{0}=M_{\mathrm{Fe}}^{\mathrm{S}}\right)$ and index $l=1 \ldots 16$ corresponds to Gd sublayers with thicknesses $t_{p}^{l}=t$ and magnetization vectors $\boldsymbol{M}_{p}^{l}$. The first term under the sum in (1) is the Zeeman energy of the layer. The second term is the effective easy-plane anisotropy with constant $K_{p}^{l}$, including demagnetizing factor ( $\boldsymbol{n}$ is a unit vector normal to the film plane). The third term is related to the exchange part of the total energy. For this energy contribution, only exchange interactions between neighbouring layers are taken into account:

$$
\begin{aligned}
& E_{p}^{0}=-J \frac{\boldsymbol{M}_{p}^{0}\left(\boldsymbol{M}_{p}^{1}+\boldsymbol{M}_{p-1}^{16}\right)}{M_{\mathrm{Fe}}^{\mathrm{S}} M_{\mathrm{Gd}}^{\mathrm{S}}}, \\
& E_{p}^{l \neq 0}=-t_{p}^{l} \lambda\left(\zeta\left(\boldsymbol{M}_{p}^{l} \boldsymbol{M}_{p}^{l+1}\right)+\zeta^{*} \frac{\left(\boldsymbol{M}_{p}^{l}\right)^{2}}{2}\right)
\end{aligned}
$$

(we bear in mind that $\boldsymbol{M}_{0}^{16} \equiv 0$ and $\boldsymbol{M}_{p}^{17} \equiv 0$ because they do not correspond to any real layers). $E_{p}^{0}$ in (2) describes AFM exchange interaction of Fe layers with neighbouring Gd sublayers (here $J$ is the coupling constant at the $\mathrm{Fe}-\mathrm{Gd}$ interface and $M_{\mathrm{Gd}}^{\mathrm{S}}$ is the saturation magnetization of $\mathrm{Gd}$ ). The exchange energy in Gd layers $E_{p}^{l}(l=1 \ldots 16)$ is described by effective field constant $\lambda$ and consists of two contributions: interaction between different atoms in the same sublayer and interaction between atoms of neighbouring sublayers. The relation between these two contributions is described by factors $\zeta$ and $\zeta^{*}$ which can be treated as fractions of nearest neighbour atoms in sublayers $l \pm 1$ and $l: \zeta=z_{l \pm 1} / z$ and $\zeta^{*}=z_{l} / z$. The total number of nearest neighbours is $z=z_{l}+z_{l-1}+z_{l+1}$ which means $\zeta^{*}=1-2 \zeta$.

In principle there could be a contribution to the total energy coming from magnetoelastic interaction [2]. However, in our model we neglect such a contribution because, according to our estimations, it does not have essential effect on static and dynamic properties of the studied sample.

In our calculations, we consider the case of a strong easy plane shape anisotropy and in-plane orientation of the magnetic field, so that static $\boldsymbol{M}_{p}^{l}$ vectors are also oriented in the film plane: $\left(\boldsymbol{M}_{p}^{l}, \boldsymbol{n}\right)=0$. This supposition is also in accordance with experimental results reported by other authors for $\mathrm{Fe} / \mathrm{Gd}$ superlattices with similar parameters [10]. To calculate static distribution of magnetization in this situation, we can write the energy (1) as a function of absolute values $M_{p}^{l}$ and angles $\varphi_{p}^{l}$ between magnetization vectors $\boldsymbol{M}_{p}^{l}$ and the external magnetic field $\boldsymbol{H}$. For given $H$ and $T$ we obtain:

$$
E_{\text {stat }}=E\left(M_{p}^{l}, \varphi_{p}^{l}\right)
$$

(we do not write the explicit expression here because it can be obtained in an obvious way). To find $M_{p}^{l}$ and $\varphi_{p}^{l}$, the following numerical iterative procedure is used. Starting from the initial approach with fixed $M_{p}^{l}$, we minimize $E_{\text {stat }}$ over angles $\varphi_{p}^{l}$. Then, at fixed newly found $\varphi_{p}^{l}$, the new values $M_{p}^{l}$ for Gd sublayers are recalculated using mean field formalism:

$$
M_{p}^{l}=M_{\mathrm{Gd}}^{\mathrm{S}} B_{s}\left(\frac{\mu H_{p}^{l}}{k_{\mathrm{B}} T}\right), \quad l=1 \ldots 16,
$$

where $B_{s}(x)$ is the Brillouin function for Gd spin $s=7 / 2$, $\mu=7.5 \mu_{\mathrm{B}}$ is the magnetic moment of the Gd ion, $\mu_{\mathrm{B}}$ is the Bohr magneton, $k_{\mathrm{B}}$ is the Boltzman constant and the effective fields $H_{p}^{l}$ are defined by derivatives of the energy (1):

$$
\boldsymbol{H}_{p}^{l}=-\frac{1}{t_{p}^{l}} \frac{\partial E}{\partial \boldsymbol{M}_{p}^{l}} .
$$

When the new $M_{p}^{l}$ values are found, we return back to minimization of the energy (3) and the procedure is repeated until the stationary self-consistent solution is found.

Depending on the initial approach, different solutions can be obtained, corresponding to different local minima of the energy. We consider the 'quasi-periodic' solution, when magnetization vectors $\boldsymbol{M}_{p}^{l}, \boldsymbol{M}_{p+1}^{l}$ are close to FM orientation. This state is in accordance with experimental results [7] and seems to provide 
global energy minimum. A more straightforward procedure to find the correct solution should minimize the free energy:

$$
F=E-T S, \quad S=\sum_{p=1}^{12} \sum_{l=1}^{16} S\left(M_{p}^{l}\right),
$$

where $S$ is the entropy of the system (mean field formulas for $S(M)$ can be found in [23]). We checked that our algorithm based on the energy minimization also provides the minimum of the free energy (6).

To compare with the experimental magnetization data, the resulting total magnetic moment per unit area of the superlattice is defined by the expression:

$$
m=\sum_{p=1}^{12} \sum_{l=0}^{16} t_{p}^{l} M_{p}^{l} \cos \varphi_{p}^{l},
$$

which takes into account the fact that the net magnetization is oriented along the magnetic field.

\subsection{Magnetization dynamics}

The magnetization dynamics is described by Landau-Lifshitz equations (LLE) with relaxation terms:

$$
\dot{\boldsymbol{M}}_{p}^{l}=-\gamma_{p}^{l}\left[\boldsymbol{M}_{p}^{l}, \boldsymbol{H}_{p}^{l}\right]+\boldsymbol{R}_{p}^{l},
$$

where $\gamma_{p}^{0}=\gamma_{\mathrm{Fe}}$ and $\gamma_{p}^{l \neq 0}=\gamma_{\mathrm{Gd}}$ are gyromagnetic ratios for $\mathrm{Fe}$ and Gd respectively. For dissipative term $\boldsymbol{R}_{p}^{l}$, we consider two contributions: 'local' and 'non-local'. The local dissipation is described by Gilbert damping:

$$
\boldsymbol{R}_{p \mathrm{G}}^{l}=\frac{\alpha_{p}^{l}}{M_{p}^{l}}\left[\boldsymbol{M}_{p}^{l}, \dot{\boldsymbol{M}}_{p}^{l}\right]
$$

where $\alpha_{p}^{0}=\alpha_{\mathrm{Fe}}$ and $\alpha_{p}^{l \neq 0}=\alpha_{\mathrm{Gd}}$ are Gilbert damping parameters for Fe and $\mathrm{Gd}$ respectively.

To describe spin diffusion effects, an additional 'non-local' damping in Gd layers is taken into account. We consider the diffusion type term proposed by Baryakhtar [24] which is written in the following form for the continual isotropic case:

$$
\boldsymbol{R}_{\mathrm{D}}=-\eta \nabla^{2} \boldsymbol{H}_{\mathrm{F}}, \quad \text { where } \quad \boldsymbol{H}_{\mathrm{F}}=-\frac{\delta F}{\delta \boldsymbol{M}} .
$$

Here $\eta$ is a phenomenological relaxation constant describing magnetic diffusion processes. For simplicity, we consider only precession motion of the vector $\boldsymbol{M}$, and disregard possible dynamical changes of its absolute value $M$. Considering only the transverse relaxation for collinear magnetic state, the term (10) can be simplified to [25]:

$$
\boldsymbol{R}_{\mathrm{D}}=-\frac{\eta}{\gamma}\left[\boldsymbol{m}, \nabla^{2} \dot{\boldsymbol{m}}\right]
$$

where $\boldsymbol{m}$ is a unit vector along $\boldsymbol{M}$. In our calculations, we take into account this simplified form of the diffusion type term. It is correct in the region of high temperatures and low fields where the collinear phase dominates, though it is not quite accurate when the twisted state is considered.

To write (11) in the discrete case, we introduce discrete (one-dimensional) analogue of Laplacian:

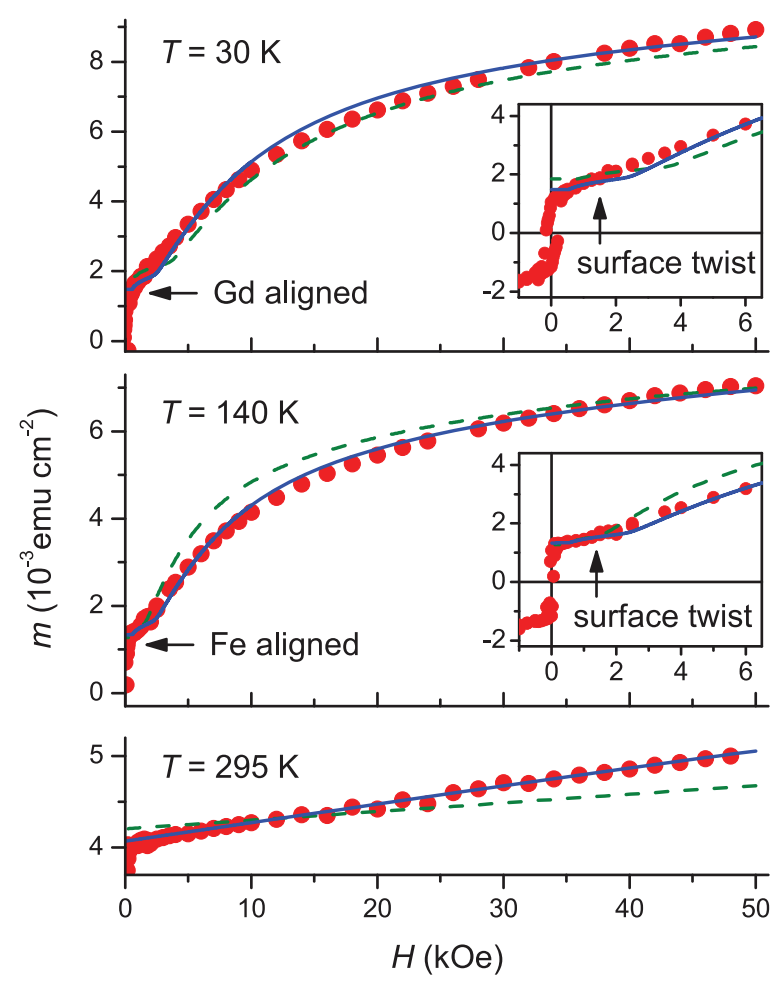

Figure 2. Magnetization curves at $T=30,140$ and $295 \mathrm{~K}$. Points are the experimental data, dashed lines correspond to their best fit with constant $\lambda$, solid lines correspond to the best fit with temperature dependent $\lambda$ (see table 2 ). Insets show low field regions with hysteresis loops.

$$
\nabla^{2} \boldsymbol{m} \rightarrow \Delta_{p}^{l} \boldsymbol{m}=\frac{\boldsymbol{m}_{p}^{l-1}-2 \boldsymbol{m}_{p}^{l}+\boldsymbol{m}_{p}^{l+1}}{t^{2}}
$$

Taking into account this definition, the considered dissipative term has the form:

$$
\boldsymbol{R}_{p \mathrm{D}}^{l}=-A^{*} M_{\mathrm{Gd}}^{\mathrm{S}}\left[\boldsymbol{m}_{p}^{l}, \Delta_{p}^{l} \dot{\boldsymbol{m}}\right], \quad l=2 \ldots 15,
$$

where we introduce the coefficient $A^{*}=\eta /\left(\gamma_{\mathrm{Gd}} M_{\mathrm{Gd}}^{\mathrm{S}}\right)$. The damping term (13) provides additional dissipation for essentially non-uniform precession modes in Gd layers. Indeed, considering the case of a flat spin wave $\boldsymbol{m}=\boldsymbol{m}_{0}+\delta \boldsymbol{m} \cdot \mathrm{e}^{\mathrm{i} \boldsymbol{k} r}$ with wave-vector $k$ in homogeneous ferromagnet, (11) transforms to Gilbert-type term with effective damping parameter $A^{*} k^{2}$ [26].

Taking into account the discussed dissipative terms, the resonance frequencies $f_{n}$ and corresponding line-widths $\Delta f_{n}$ are calculated as real and imaginary parts of complex eigenvalues $\omega_{n}$ of the system (8) linearized near static $\boldsymbol{M}_{p}^{l}$ position:

$$
f_{n}=\operatorname{Re}\left(\omega_{n}\right) / 2 \pi, \quad \Delta f_{n}=2 \cdot \operatorname{Im}\left(\omega_{n}\right) / 2 \pi,
$$

where $n$ is the resonance mode number.

\section{Results and discussion}

\subsection{Static magnetization}

Figure 2 shows experimental magnetization curves at different temperatures and the result of their approximation within the mean field model in two cases. First, the best least-squares fit of $m(H)$ curves was obtained considering constant $\lambda$ (fit 1). 
Table 2. Fitting parameters of magnetization curves (figure 2) in the case when $\lambda$ is considered as constant (fit 1) and in the case when $\lambda$ differs for different temperatures (fit 2).

\begin{tabular}{llllll}
\hline & $\begin{array}{l}M_{\mathrm{Fe}}^{\mathrm{S}} \\
\left(\mathrm{emu} \mathrm{cm}^{-3}\right)\end{array}$ & $\begin{array}{l}M_{\mathrm{Gd}}^{\mathrm{S}} \\
\left(\mathrm{erg} \mathrm{cm}^{-2}\right)\end{array}$ & $\zeta$ & $\lambda$ \\
\hline fit 1 & 1230 & 1150 & -44 & 0.22 & 630 \\
\hline & & & & & $380(30 \mathrm{~K})$ \\
fit 2 & 1270 & 1150 & -39 & 0.33 & $\begin{array}{l}630(140 \mathrm{~K}) \\
960(295 \mathrm{~K})\end{array}$ \\
\hline
\end{tabular}

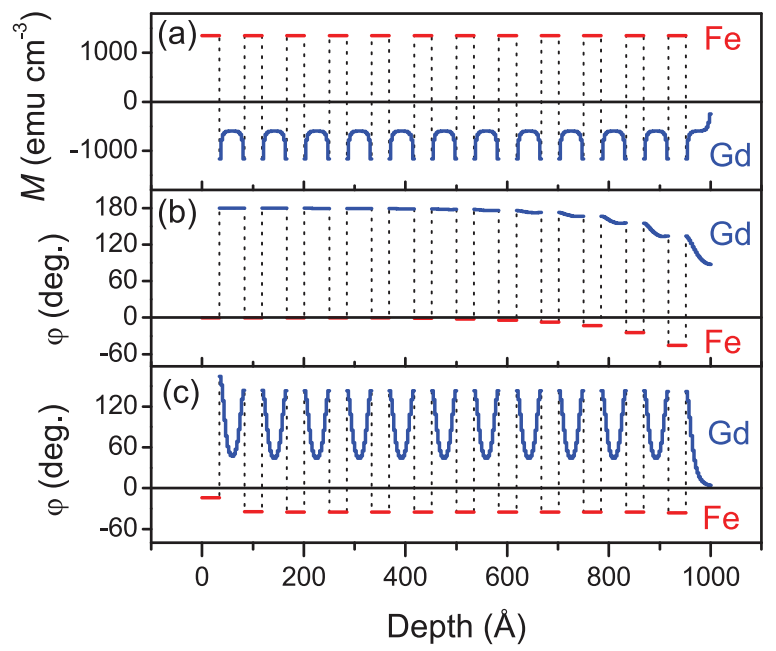

Figure 3. Calculated magnetization profiles at $T=140 \mathrm{~K}$ for different magnetic fields: (a) $H=0.1$ kOe corresponding to $\mathrm{Fe}$-aligned state (magnetization component along the magnetic field is shown), (b) $H=1 \mathrm{kOe}$ corresponding to surface twist state and (c) $H=10 \mathrm{kOe}$ corresponding to twisted state (the angle between the magnetization vector and the magnetic field is shown).

Then, we tried to achieve a better fit taking into account temperature dependence of the effective field parameter $\lambda$ (fit 2). The resulting fitting parameters are shown in table 2.

In both cases the found $M_{\mathrm{Fe}}^{\mathrm{S}}, M_{\mathrm{Gd}}^{\mathrm{S}}$ and $J$ values are close to each other. The obtained saturation magnetization values for $\mathrm{Fe}$ and $\mathrm{Gd}$ are noticeably smaller than their bulk values $\left(M_{\mathrm{Fe}}^{\text {bulk }} \approx 1750, M_{\mathrm{Gd}}^{\text {bulk }} \approx 2020 \mathrm{emu} \mathrm{cm}^{-3}\right)$. Partially, this effect can be ascribed to imperfections of $\mathrm{Fe}-\mathrm{Gd}$ interfaces and intermixing of $\mathrm{Fe}$ and $\mathrm{Gd}$ atoms at them. However, the quality of interfaces is rather good in our case (see table 1). Thus, the most probable reason for the observed strong reduction of magnetization seems to be due to a large degree of structural disorder and amorphousness of the grown FM layers. Indeed, such effects were previously reported for both thin polycrystalline Gd [27, 28] and amorphous Fe layers [29]. In both cases the magnetization reduction can reach $\sim 50 \%$ of its bulk value.

The obtained interlayer AFM exchange energy $J \approx-40$ erg $\mathrm{cm}^{-2}$ recalculated per one interfacial atom gives approximately $J \approx-0.02 \mathrm{eV} \approx-200 \mathrm{~K}$ which is in accordance with [6]. The parameter $\zeta$ differs 1.5 times for fit 1 and fit 2 . The value $\zeta \approx 0.33$ obtained in fit 2 means $z_{l} \approx z_{l \pm 1} \approx z / 3$ which seems reasonable.

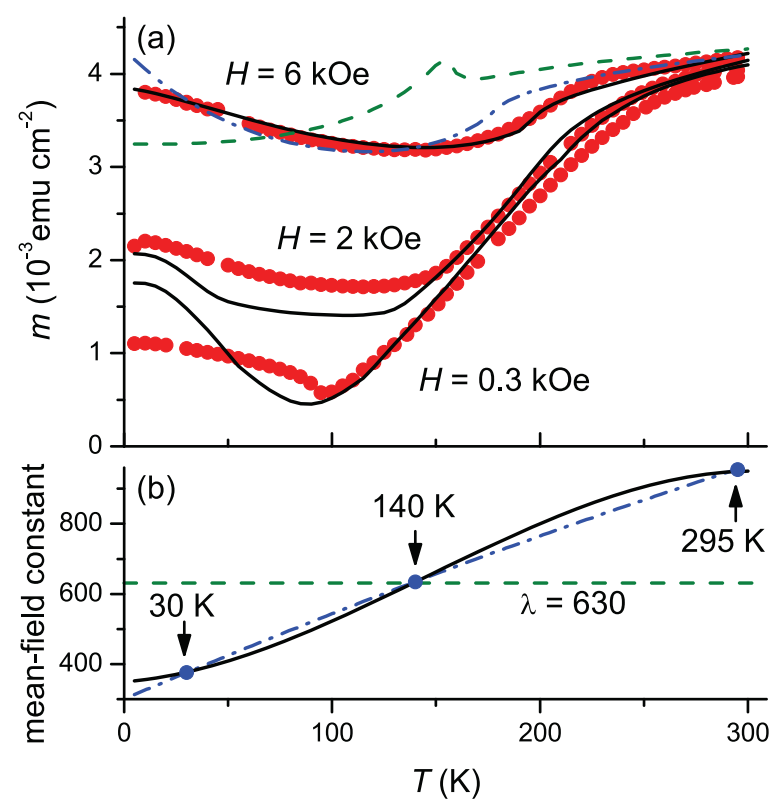

Figure 4. Magnetic moment per unit area as a function of temperature measured at $H=0.3,2$ and $6 \mathrm{kOe}$ (a). Points are the experimental data, lines are theoretical calculations with different $\lambda(T)$ dependences (b). The dashed line corresponds to constant $\lambda$, the dash-dotted and solid lines correspond to the second- and thirdorder curves $\lambda(T)$ respectively.

Approximation of the experimental data with temperature dependent $\lambda$ provides much better quantitative agreement. The model predicts all the observed peculiarities of magnetization curves. In low fields $(H \lesssim 0.1 \mathrm{kOe})$, a collinear magnetic state is realized: 'Gd-aligned' at low temperatures $(T=30 \mathrm{~K})$ and 'Fe-aligned' at high temperatures $(T=140$, $295 \mathrm{~K})$. Increasing the magnetic field up to $H \approx 2 \mathrm{kOe}$ at $T=30,140 \mathrm{~K}$, we observe a relatively weak increase of sample magnetization which is associated with a distortion of the collinear state near superlattice surface ('surface twist' state). Above $H \approx 2 \mathrm{kOe}$ the magnetization increases more rapidly due to a transition of the superlattice to the twisted state. The discussed effect is more pronounced at $T=140 \mathrm{~K}$ while at $T=30 \mathrm{~K}$ it is probably obscured by magnetic domains due to increased anisotropy in Gd. Examples of calculated magnetization depth profiles for different magnetic states are shown in figure 3 .

Figure 4 demonstrates experimental and calculated $m(T)$ curves obtained at different magnetic fields. It is clearly seen, that the experimental data can not be approximated well assuming the constant value of the effective field parameter. The $m(T)$ curve at $H=6 \mathrm{kOe}$ is described much better if we consider a second order curve for $\lambda(T)$, passing through the three points $\lambda(30 \mathrm{~K}), \lambda(140 \mathrm{~K}), \lambda(295 \mathrm{~K})$ determined above from fitting of the magnetization curves. An almost ideal approximation for $H=6 \mathrm{kOe}$ can be achieved using a third order polynomial:

$$
\lambda(T) \approx 800+505 \tau-255 \tau^{2}-310 \tau^{3},
$$

where $\tau=\left(T-T_{\mathrm{C}}\right) / T_{\mathrm{C}}$ with gadolinium Curie temperature $T_{\mathrm{C}} \approx 200 \mathrm{~K}$ defined from the mean-field relation: 


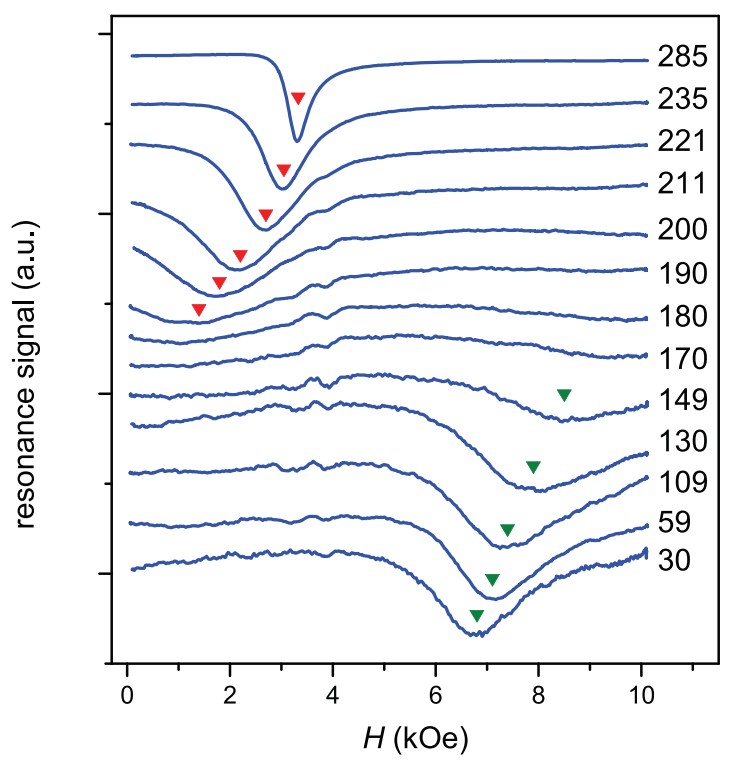

Figure 5. Examples of resonance spectra at $f=25.9 \mathrm{GHz}$ and different temperatures (30-285 K) shown in the plot. Two resonance modes are shown by arrows.

$$
\lambda\left(T_{\mathrm{C}}\right)=\frac{3 k_{\mathrm{B}} T_{\mathrm{C}}}{\mu M_{\mathrm{Gd}}^{\mathrm{S}}} \frac{s}{s+1} .
$$

The obtained $T_{\mathrm{C}}$ value is in agreement with [6].

The presence of a phase transition at $T \approx 200 \mathrm{~K}$ is clearly seen as a kink on the $m(T)$ curve at $H=6 \mathrm{kOe}$ where a transition from twisted phase to Fe-aligned phase occurs. In the case of the weak field $H=0.3 \mathrm{kOe}$, the Fe-aligned phase is realized at $T \gtrsim 100 \mathrm{~K}$. In this situation we do not observe essential peculiarities on the $m(T)$ curve at $T_{\mathrm{C}}$ because of a strong exchange interaction at $\mathrm{Fe}-\mathrm{Gd}$ interfaces. This interaction can be treated as an additional effective field acting on Gd layers which stimulates the FM moment above $T_{\mathrm{C}}$ and obscures the ferromagnetic phase transition. As a result, we observe a relatively smooth increase of the total superlattice magnetization with temperature, without a pronounced peculiarity at $T_{\mathrm{C}}$. For the intermediate field $H=2 \mathrm{kOe}$, our calculations predict an existence of the surface twist state in the temperature range $130-200 \mathrm{~K}$ while the twisted state is realized below $130 \mathrm{~K}$. In this case we also observe only a weak kink on the $m(T)$ curve as the structure transforms to collinear Fe-aligned state above $T_{\mathrm{C}}=200 \mathrm{~K}$.

In the region of low temperatures and fields, the role of domain structure is probably important and the accordance between the experiment and the model calculation is not perfect (figure 4(a)). Nevertheless, the calculated compensation temperatures $T_{\text {comp }} \approx 90 \mathrm{~K}$ is in a good agreement with the experimental value.

\subsection{Ferromagnetic resonance}

Examples of FMR spectra obtained at fixed frequency and different temperatures are shown in figure 5. The sample demonstrates two Lorentz-shaped absorption lines. One narrow peak is observed at room temperature. As temperature decreases,
Table 3. Parameters used for FMR modelling.

\begin{tabular}{llllll}
\hline$\gamma_{\mathrm{Fe}} / 2 \pi$ & $\gamma_{\mathrm{Gd}} / 2 \pi$ \\
$\left(\mathrm{GHz} \mathrm{kOe}^{-1}\right)$ & $K$ & $\alpha_{\mathrm{Fe}}$ & $\alpha_{\mathrm{Gd}}$ & $\begin{array}{l}A^{*} \\
\left(\mathrm{~nm}^{2}\right)\end{array}$ \\
\hline 2.94 & 2.80 & 12.6 & 0.01 & 0.02 & 0.025 \\
\hline
\end{tabular}
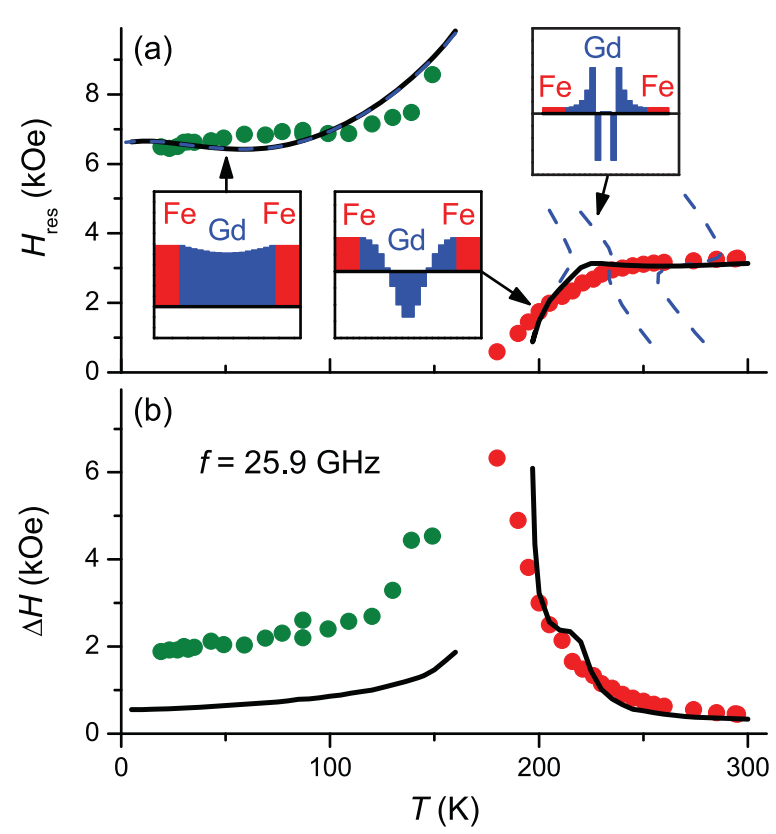

Figure 6. Resonance field (a) and linewidth (b) as a function of temperature at $f=25.9 \mathrm{GHz}$. Points are the experimental data, lines are the result of modelling. Dashed lines correspond to the case when only local Gilbert damping is taken into account. For solid lines, the additional non-local diffusion-type dissipative term in LLE is considered. Insets show calculated precession profiles (real part of eigenvectors) for different modes normalized on static magnetization distribution.

it broadens and shifts towards lower fields. The second peak observed at low temperatures shifts towards higher fields at heating. The resulting temperature dependencies of resonance field $H_{\text {res }}(T)$ and linewidth $\Delta H(T)$ for two lines at frequency $25.9 \mathrm{GHz}$ are shown in figure 6.

Examples of frequency vs field dependencies, $f(H)$, at different temperatures are demonstrated in figure 7 . For both absorption lines, monotonically increasing $f(H)$ dependencies are observed.

The calculations of eigenfrequencies in the system were performed using model parameters obtained from static magnetization data (with temperature dependent $\lambda$ ). The dynamic characteristics are shown in table 3 . For gyromagnetic ratio in $\mathrm{Fe}$ and Gd layers, we used the values for bulk Fe and Gd. The effective easy-plane anisotropy constant $K$ had the same value for Fe and Gd layers corresponding to the shape anisotropy of a thin FM film (demagnetization factor $4 \pi$ ). In particular, this anisotropy leads to the in-plane orientation of static magnetization for both $\mathrm{Fe}$ and Gd layers. For the local Gilbert damping parameters, we considered realistic values [30]. The non-local damping parameter $A^{*}$ was estimated from the FMR linewidth (more discussions are below). As a first approximation, we neglected a possible temperature dependence of the damping parameters. 

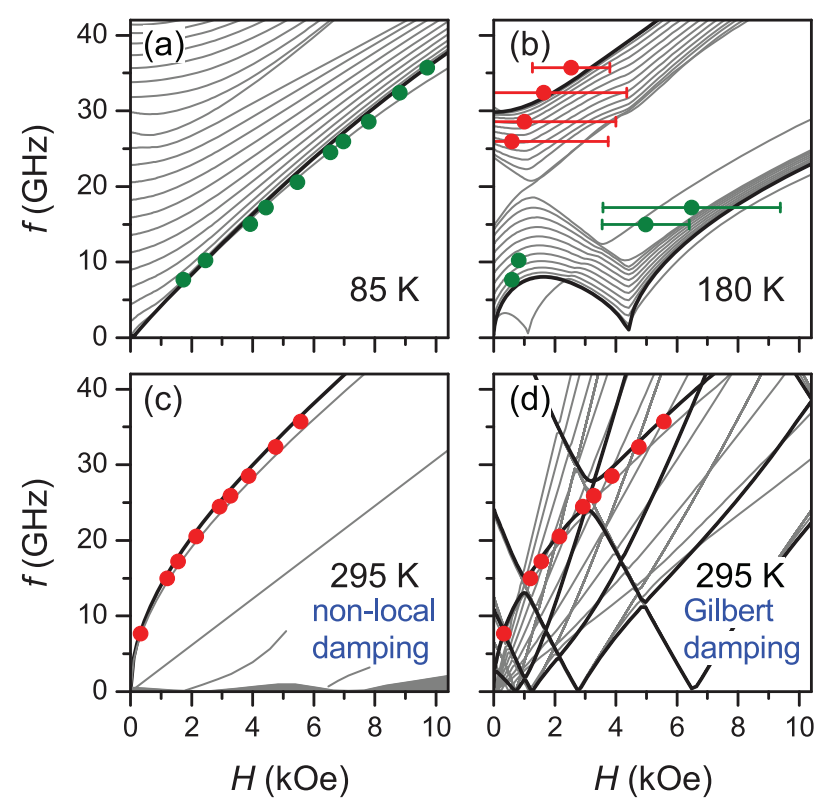

Figure 7. Resonance frequency as a function of applied field at different temperatures. Points are the experimental data, lines are the result of modelling. The thin lines correspond to calculated eigenfrequencies for the complete multilayer $[\mathrm{Fe} / \mathrm{Gd}]_{12}$. The thick lines are the result of modelling of one period of the superlattice. For illustrative purposes, only the calculated modes with relatively small dissipation $(\Delta f<10 \mathrm{GHz})$ are shown. The theoretical spectra ((a)-(c)) are obtained taking into account the non-local damping term in LLE. As a comparison, the room-temperature spectrum calculated without this term is shown (d).

The complete calculated spectrum of eigenfrequencies for the $[\mathrm{Fe} / \mathrm{Gd}]_{12}$ structure consists of a large number of resonance branches $f(H)$ corresponding to standing spin-waves within the superlattice (figure 7). Experimentally, we observe only two resonance lines which are obviously associated with the most homogeneous precession modes. For this reason, we performed a modelling for one period of the superlattice $\mathrm{Fe}\left(t_{\mathrm{Fe}} / 2\right) / \mathrm{Gd}\left(t_{\mathrm{Gd}}\right) / \mathrm{Fe}\left(t_{\mathrm{Fe}} / 2\right)$ to calculate the eigenfrequencies corresponding to in-phase precession of the neighbouring $\mathrm{Fe}$ layers. Figure 7 demonstrates that this method allows identification of such modes among the resonance branches in the whole superlattice.

We used two approaches to modeling magnetic dynamics in the superlattice. The first one considers only Gilbert damping in each magnetic sublayer, while the second one also takes into account the non-local damping described by the term (13) in LLE. It must be noted that in our situation, the introduction of damping terms into LLE did not have much effect on the real part of eigenfrequencies in the region of low temperatures $(T \lesssim 200 \mathrm{~K})$. In contrast, the spectrum at high temperatures $(T \gtrsim 200 \mathrm{~K}$ ) is strongly dependent on the used damping terms.

Figure 6(a) demonstrates a comparison of the experimental and calculated $H_{\text {res }}(T)$ dependences. It is seen that the high temperature part of the $H_{\text {res }}(T)$ curve is poorly described when only the Gilbert damping term in LLE is considered. In this case, the form of the $H_{\text {res }}(T)$ dependence is strongly deformed by the presence of high-order spin-wave modes within the Gd layer. These modes are crossing with each other forming the complicated spectrum due to the effect of mode repulsion, while the experiment shows simple monotonic $H_{\text {res }}(T)$ dependence. This discrepancy can also be seen comparing the experimental and calculated $f(H)$ dependences when only Gilbert damping is taken into account (figure 7(d)).

Adding the non-local damping term to LLE changes the situation drastically. This term suppresses the high-order spinwave modes in the Gd layer and the resulting high-temperature branch of the $H_{\text {res }}(T)$ dependence is described sufficiently well in the frame of the considered model (figure 6). Similarly, the experimental high-temperature $f(H)$ dependences are better described taking into account the non-local damping (figure 7(c)).

Comparing the experimental spectra with model results, the types of precession modes for the observed resonance lines can be identified. The line at low temperatures (figures 5 and 6) corresponds to the low-frequency branch of the spectrum (figures 7(a) and (b)). This mode is associated with the in-phase precession of Fe and Gd layers (see the inset in figure 6). The line observed at high temperatures corresponds to the high-frequency branch of the spectrum (figures 7(b) and (c)). For this spectral branch, the precession phase of the central part of the Gd layer is opposite the precession phase of the Fe layers.

Let us discuss in more detail the evolution of the two spectral branches with temperature. The $f(H)$ dependencies for both modes are presented in figure 7(b) at $T=180 \mathrm{~K}$. The low-frequency branch $f(H)$ calculated for one period of the superlattice demonstrates a critical field $H \approx 4 \mathrm{kOe}$ where the eigenfrequency vanishes. This field corresponds to a phase transition of the structure from the collinear Fe-aligned state to the twisted state. (Strictly speaking, when the complete superlattice is considered, a weak surface twist takes place below $H \approx 4 \mathrm{kOe}$, while the real transition to the collinear state occurs below $H \approx 1 \mathrm{kOe}$.) As temperature rises, the magnetization of Gd decreases stabilizing the Fe-aligned phase. Thus, the phase transition to the twisted state is shifted to higher fields. As a consequence, the resonance field is increasing with temperature and the effect is observed experimentally (the low-temperature branch in figure 6).

The high-frequency branch $f(H)$ is an 'exchange' mode. It demonstrates a gap in the spectrum at $H=0$. The value of this gap can be roughly considered to be proportional to the exchange stiffness of Gd layers. As temperature rises, the $\mathrm{Gd}$ exchange stiffness diminishes and the gap in the spectrum decreases. While measuring the FMR spectra at constant frequency, the resonance line must appear at some temperature in zero field and shift to higher fields at increasing temperature. This situation is observed experimentally for the high-temperature branch in figure 6.

The correspondence between experimental and calculated dependencies $f(H)$ is good in the limit of high and low temperatures (figures 7(a) and (c)). In the vicinity of Curie temperature of Gd the agreement becomes worse (figure 7(b)) which fact is probably due to higher sensitivity of the calculated spectrum to the model parameters. Nevertheless, taking into account the simplicity of the model, the general correspondence between experiment and theory for both $f(H)$ and $H_{\text {res }}(T)$ dependencies is reasonable. 
The considered non-local damping term allows a qualitative description of the linewidth behaviour. The calculated temperature dependence of the linewidth $\Delta H(T)$ estimated by formula $\Delta H \approx \Delta f \cdot\left(\mathrm{d} H_{\text {res }} / \mathrm{d} f\right)$ is shown in figure $6(\mathrm{~b})$. The experimental $\Delta H(T)$ dependence for the high-temperature branch of the spectrum can be approximated well within the considered model with damping parameters shown in table 3 . For the low-temperature branch, the correspondence is not so good. One of the reasons for this could be the limited applicability of the simplified form of the non-local damping term used (11). Strictly speaking, this form is correct only when the collinear phase is considered which is the case for the high-temperature spectral branch in figure 6 . In contrast, the low-temperature branch in figure 6 corresponds to the twisted state where more complicated diffusion type terms must be taken into account [25]. Of course, other mechanisms of line broadening cannot be excluded. In particular, the polycrystallinity of the Gd layers may have significant influence on the line-width at low temperatures.

\subsection{Discussion}

As can be seen from our analysis, the simple molecular field approach to modelling the Fe/Gd superlattice does not provide good approximation of the experimental results. Nevertheless, a formal supposition of a temperature dependent mean-field parameter seems to be productive and leads to good approximation of both static and dynamic magnetic properties of the sample. A physical basis for such a supposition may become clearer if we consider improved effective field approaches.

In contrast to the molecular field theory where the individual spins are considered as statistically independent, the constant coupling approximation [12] takes into account pair correlations of the nearest neighbour spins. Within this theory, the exchange energy can also be treated in terms of the effective field $H_{\mathrm{ex}}$ acting on the spin. However, this effective field has much more complex form $H_{\mathrm{ex}}=\Phi(T, M)$ as compared the case of the molecular field approximation $H_{\mathrm{ex}}=\lambda M$. A relatively simple expression for $H_{\mathrm{ex}}$ can be obtained in the case of homogeneous ferromagnet with spin $1 / 2$. In the limit of small magnetization $M \ll M_{S}$, it has the form $H_{\mathrm{ex}} \approx \lambda(T) M$ with

$$
\lambda(T) \sim \frac{k_{\mathrm{B}} T}{2 J_{1}}\left[1-\exp \left(-\frac{2 J_{1}}{k_{\mathrm{B}} T}\right)\right],
$$

where $J_{1}$ is the exchange energy between nearest neighbour spins. The approximation (17) is valid for $T \gg T_{\mathrm{C}}$. However, it is roughly true even for $T \sim T_{\mathrm{C}}$.

According to (17), $\lambda(T)$ is an increasing function of temperature that is in qualitative agreement with the experimental result. However, to make a quantitative comparison, more detailed theoretical microscopic analysis is needed, taking into account the real Gd spin value and considering the complex magnetic structure within Gd layers.

Besides the static magnetization data, FMR results are also described relatively well using the proposed approach. However, as shown, the additional non-local damping (11) in LLE must be considered for a better description of the resonance spectra.
Recently, the dissipative term (11) was found to be important to describe magnetization dynamics in thin films and multilayers based on FM metals [20, 21, 26]. Physically, this term is attributed to dissipative spin currents within metallic ferromagnet. The value of damping coefficient $A^{*}$ found in [26] for transition ferromagnetic metals is estimated as $A^{*} \sim 0.1 \mathrm{~nm}^{2}$. In our case of Gd layers, the non-local damping parameter $A^{*}$ is about four times smaller (see table 3 ). The reason for this could be due to the different character of magnetism in rear-earth $4 f$-metals. In contrast to transition $3 d$-metals, the rear-earth $4 f$-metals demonstrate much weaker spin polarization of itinerant electrons being coupled relatively weakly with the spins of localized $4 f$ electrons [31]. Thus, the effects of spin diffusion in $\mathrm{Gd}$ are expected to be much weaker than those in transition $3 d$-metals.

\section{Conclusion}

In this work, the experimentally obtained magnetization curves and FMR spectra of the Fe/Gd superlattice were analysed in the frame of mean field approximation in the wide range of temperatures 5-295 K. As a consequence, we would like to point out two main results.

First, we found that both static and dynamic magnetic properties of the sample can be described well by the mean field model only if we suppose a significant temperature dependence of the mean field parameter in Gd layers. Second, the FMR data clearly demonstrated the importance of non-local damping in Gd layers to describe the magnetization dynamics in the multilayer.

In the frame of our phenomenological analysis, we cannot tell with certainty about the microscopic origin of these effects. The experimentally observed temperature dependence of the mean field parameter obviously indicates limited applicability of the simple Weiss molecular field approach in the considered case. The reasons for this can be both structural imperfections of the real sample and insufficient accuracy of the mean field approximation. In the latter case, the constant coupling approximation could probably provide better description of the experimental results. However, a modification of this theory is needed to be applied to the considered $\mathrm{Fe} / \mathrm{Gd}$ system where complicated magnetic order types are realized.

A possible reason for the observed effect of non-local damping could be due to dissipative spin currents in $\mathrm{Gd}$ layers. The obtained value of the non-local damping in $\mathrm{Gd}$ is noticeably smaller than that for transition metals. This result can indicate relatively weak spin diffusion effects in rare-earth metals.

\section{Acknowledgments}

X-ray measurements were performed at the Collective Use Center of IMP. We would like to thank A Mukhin, V Ivanov and A Kuz'menko (GPI RAS) for assistance in performing measurements on a SQUID magnetometer. We are grateful to D Kholin and V Marchenko (IPP RAS) for useful discussions 
of our work. The work is partially supported by the Russian Foundation for Basic Research (grants No.15-07-01170, No. 16-02-00061 and No. 14-22-01063) and by the Ministry of Education and Science of the Russian Federation (grant No. 14.Z50.31.0025).

\section{References}

[1] Camley R E 2015 Thermal properties of magnetic multilayers and nanostructures: applications to static and dynamic behavior Magnetism of Surfaces, Interfaces, and Nanoscale Materials (Handbook of Surface Science vol 5) ed R E Camley et al (Amsterdam: North-Holland) Chapter 6

[2] Camley R E and Stamps R L 1993 J. Phys.: Condens. Matter 53727

[3] Ishimatsu N, Hashizume H, Hamada S, Hosoito N, Nelson C S, Venkataraman C T, Srajer G and Lang J C 1999 Phys. Rev. B 609596

[4] Hosoito N, Hashizume H, Ishimatsu N, Bae I-T, Srajer G, Lang J C, Venkataraman C T and Nelson C S 2002 Japan J. Appl. Phys. 411331

[5] Haskel D, Srajer G, Lang J C, Pollmann J, Nelson C S, Jiang J S and Bader S D 2001 Phys. Rev. Lett. 87207201

[6] Choi Y, Haskel D, Camley R E, Lee D R, Lang J C, Srajer G, Jiang J S and Bader S D 2004 Phys. Rev. B 70134420

[7] Kravtsov E, Haskel D, te Velthuis S G E, Jiang J S and Kirby B J 2009 Phys. Rev. B 79134438

[8] Camley R E 1987 Phys. Rev. B 353608

[9] Takanashi K, Kamiguchi Y, Fujimori H and Motokawa M 1992 J. Phys. Soc. Japan 613721

[10] Hosoito N, Hashizume H and Ishimatsu N 2002 J. Phys.: Condens. Matter 145289

[11] Smart J S 1966 Effective Field Theories of Magnetism (Philadelphia, PA: Saunders)

[12] Kasteleijn P W and Van Kranendonk J 1956 Physica XXII 317

[13] Mangin S et al 2014 Nat. Mater. 13286
[14] Xu C, Ostler T A and Chantrell R W 2016 Phys. Rev. B 93054302

[15] Drovosekov A B et al 2015 J. Exp. Theor. Phys. 1201041

[16] Demirtas S, Harward I, Camley R E, Celinski Z, Hossu M R, Koymen A R, Yu C and Pechan M J 2010 arXiv:1002.4889

[17] Svalov A V, Barandiarán J M, Vas'kovskiy V O, Kurlyandskaya G V, Lezama L, Bebenin N G, Gutiérrez J and Schmool D S 2001 Chin. Phys. Lett. 18973

[18] Patrin G S, Vas'kovskii V O, Svalov A V, Eremin E V, Panova M A and Vasil'ev V N 2006 J. Exp. Theor. Phys. 102131

[19] Montoya E, Sebastian T, Schultheiss H, Heinrich B, Camley R E and Celinski Z 2015 Magnetization dynamics Magnetism of Surfaces, Interfaces, and Nanoscale Materials (Handbook of Surface Science vol 5) ed R E Camley et al (Amsterdam: North-Holland) Chapter 3

[20] Tserkovnyak Y, Hankiewicz E M and Vignale G 2009 Phys. Rev. B 79094415

[21] Nembach H T, Shaw J M, Boone C T and Silva T J 2013 Phys. Rev. Lett. 110117201

[22] Landes J, Sauer Ch, Kabius B and Zinn W 1991 Phys. Rev. B 448342

[23] Tuszyński J A and Wierzbicki W 1991 Am. J. Phys. 59555

[24] Bar'yakhtar V G 1984 Sov. Phys. JETP 60863

[25] Wang W et al 2015 Phys. Rev. B 92054430

[26] Li Y and Bailey W E 2016 Phys. Rev. Lett. 116117602

[27] Romera M, Muñoz M, Maicas M, Michalik J M, de Teresa J M, Magén C and Prieto J L 2011 Phys. Rev. B 84094456

[28] Ward C, Scheunert G, Hendren W R, Hardeman R, Gubbins M A and Bowman R M 2013 Appl. Phys. Lett. 102092403

[29] Handschuh S, Landes J, Köbler U, Sauer Ch, Kisters G, Fuss A and Zinn W 1993 J. Magn. Magn. Mater. 119254

[30] Platow W, Anisimov A N, Dunifer G L, Farle M and Baberschke K 1998 Phys. Rev. B 585611

[31] Frietsch B, Bowlan J, Carley R, Teichmann M, Wienholdt S, Hinzke D, Nowak U, Carva K, Oppeneer P M and Weinelt M 2015 Nat. Commun. 68262 